\title{
Usefulness of Magnetic Particle Imaging for Predicting the Therapeutic Effect of Magnetic Hyperthermia
}

\author{
Kenya Murase, Marina Aoki, Natsuo Banura, Kohei Nishimoto, Atsushi Mimura, \\ Tomomi Kuboyabu, Isamu Yabata \\ Department of Medical Physics and Engineering, Division of Medical Technology and Science, Faculty of Health \\ Science, Graduate School of Medicine, Osaka University, Osaka, Japan \\ Email: murase@sahs.med.osaka-u.ac.jp
}

Received 28 May 2015; accepted 23 June 2015; published 26 June 2015

Copyright (C) 2015 by authors and Scientific Research Publishing Inc.

This work is licensed under the Creative Commons Attribution International License (CC BY). http://creativecommons.org/licenses/by/4.0/

(c) (i) Open Access

\section{Abstract}

Purpose: To investigate the usefulness of magnetic particle imaging (MPI) for predicting the therapeutic effect of magnetic hyperthermia (MH). Materials and Methods: First, we performed phantom experiments to investigate the relationship between the MPI value and the temperature rise of magnetic nanoparticles (MNPs) under an alternating magnetic field (AMF). The MPI value was defined as the pixel value of the transverse image reconstructed from the third-harmonic signals. Samples filled with various iron concentrations of MNPs (Resovist $\left.{ }^{\circledR}\right)$ were prepared and were imaged using our MPI scanner. These samples were also heated using the AMF, and the specific loss power (SLP) and volume-specific loss power (vSLP) were calculated from the initial slope of the time-dependent temperature rise. Second, we performed animal experiments using tumor-bearing mice, which were divided into untreated $(n=10)$ and treated groups $(n=20)$. The tumors in the treated group were injected with Resovist ${ }^{\circledR}$ at an iron concentration of $250 \mathrm{mM}(\mathrm{n}=10)$ or 500 $\mathrm{mM}(\mathrm{n}=10)$, and received MH for $20 \mathrm{~min}$, during which the temperatures in the tumor and rectum were measured. The relative tumor volume growth (RTVG) was calculated from $\left(V_{15}-V_{0}\right) / V_{0}$, where $V_{0}$ and $V_{15}$ represented the tumor volume on day 0 and day 15 after $\mathrm{MH}$, respectively. Results: In phantom experiments, the MPI value had significant correlations with the iron concentration of MNPs $(r=0.997)$, temperature rise $(r=0.981)$, and vSLP $(r=0.961)$. In animal experiments, the MPI value had significant correlations with the temperature rise in the tumor $(r=0.731)$ and RTVG ( $r=-0.687)$. Conclusion: Our preliminary results suggest that MPI is useful for predicting the therapeutic effect of $\mathrm{MH}$.

\section{Keywords}

Magnetic Particle Imaging, Magnetic Hyperthermia, Magnetic Nanoparticles, Phantom

How to cite this paper: Murase, K., Aoki, M., Banura, N., Nishimoto, K., Mimura, A., Kuboyabu, T. and Yabata, I. (2015) Usefulness of Magnetic Particle Imaging for Predicting the Therapeutic Effect of Magnetic Hyperthermia. Open Journal of Medical Imaging, 5, 85-99. http://dx.doi.org/10.4236/ojmi.2015.52013 


\section{Experiments, Animal Experiments}

\section{Introduction}

Hyperthermia is one of the promising approaches to cancer therapy. The most commonly used heating method in the clinical setting is capacitive heating that uses a radiofrequency (RF) electric field [1]. However, a major technical problem with hyperthermia is the difficulty of heating the targeted tumor to the desired temperature without damaging the surrounding tissues, as the electromagnetic energy must be directed from an external source and must penetrate normal tissue. Other hyperthermia modalities, including RF ablation and ultrasound hyperthermia, have been reported [2] [3], but the efficacy of these modalities depends on the size and depth of the tumor, and disadvantages include limited ability to target the tumor and control the exposure.

Hyperthermia with the use of magnetic nanoparticles (MNPs) (magnetic hyperthermia) was developed in the 1950s [4] and is still under development in the effort to overcome the above disadvantages [5]-[7]. MNPs generate heat in an alternating magnetic field as a result of hysteresis and relaxational losses, which results in heating of the tissue in which MNPs accumulate [8] [9]. With the development of precise methods for synthesizing functionalized MNPs [10] [11], MNPs with functionalized surfaces, which have high specificity for tumor tissue, have been developed as heating elements for magnetic hyperthermia [12]. Furthermore, there is renewed interest in magnetic hyperthermia as a treatment modality for cancer, especially when it is combined with other more traditional therapeutic approaches such as the co-delivery of anticancer drugs or photodynamic therapy [13]. From these aspects, magnetic hyperthermia has received much recent attention.

Accurate knowledge of the distribution and quantity of MNPs within the targeted tumor is crucial for effective and safe treatment planning of magnetic hyperthermia [13]. Recently, a micro computed tomography (micro CT) imaging technique was proposed as a noninvasive and nondestructive method for investigating the distribution of MNPs in tumors [14]. The use of a high-resolution micro CT will be promising and useful for analyzing the deposition of MNPs and temperature elevation in the tumors. To the best of our knowledge, however, the studies using micro CT [14] are limited to ex vivo studies. Thus, the development of a readily available, noninvasive, and quantitative in vivo imaging technique will be necessary if magnetic hyperthermia treatment is to succeed.

Recently, a new imaging method called magnetic particle imaging (MPI) has been introduced [15]. MPI allows imaging of the spatial distribution of MNPs with high sensitivity, spatial resolution, and imaging speed [15]. MPI uses the nonlinear response of MNPs to detect their presence in an alternating magnetic field, which is referred to here as the drive magnetic field. Spatial encoding is accomplished by saturating the MNPs over most of the imaged region using a static magnetic field (selection magnetic field), except in the vicinity of a special position called the field-free point [15] or field-free line [16]. We have developed a system for MPI with a field-free line encoding scheme, in which the field-free line is generated using two opposing neodymium magnets, and transverse images are reconstructed from the third-harmonic signals received by a gradiometer coil using the maximum likelihood-expectation maximization (ML-EM) algorithm [17] [18].

The purpose of this study was to investigate the usefulness of MPI for predicting the therapeutic effect of magnetic hyperthermia treatment using phantom and in vivo small animal experiments.

\section{Materials and Methods}

\subsection{System for Magnetic Particle Imaging}

The details of our MPI system are described in our previous paper [17]-[20]. In brief, a drive magnetic field was generated using an excitation coil (solenoid coil $100 \mathrm{~mm}$ in length, $80 \mathrm{~mm}$ in inner diameter, and $110 \mathrm{~mm}$ in outer diameter). AC power was supplied to the excitation coil by a programmable power supply (EC1000S, NF Co., Yokohama, Japan), and was controlled using a sinusoidal wave generated by a digital function generator (DF1906, NF Co., Yokohama, Japan). The frequency and peak-to-peak strength of the drive magnetic field were taken as $400 \mathrm{~Hz}$ and $20 \mathrm{mT}$, respectively. The signal generated by MNPs was received by a gradiometer coil (50 $\mathrm{mm}$ in length, $35 \mathrm{~mm}$ in inner diameter, and $40 \mathrm{~mm}$ in outer diameter), and the third-harmonic signal was extracted using a preamplifier (T-AMP03HC, Turtle Industry Co., Ibaragi, Japan) and a lock-in amplifier (LI5640, 
NF Co., Yokohama, Japan). The output of the lock-in amplifier was converted to digital data by a personal computer connected to a multifunction data acquisition device with a universal serial bus port (USB-6212, National Instruments Co., TX, USA). The sampling time was taken as $10 \mathrm{~ms}$. When measuring signals using the gradiometer coil, a sample was placed $12.5 \mathrm{~mm}$ (i.e., one quarter of the coil length) from the center of the gradiometer coil and the coil, including the sample, was moved such that the center of the sample coincided with the position of the field-free line. The selection magnetic field was generated by two opposing neodymium magnets (Neomax Engineering Co., Gunma, Japan). The field-free line can be generated at the center of the two neodymium magnets.

To acquire projection data for image reconstruction, a sample (phantom or mouse) in the receiving coil was automatically rotated around the z-axis over $180^{\circ}$ in steps of $5^{\circ}$ and translated in the $\mathrm{x}$-direction from $-16 \mathrm{~mm}$ to $16 \mathrm{~mm}$ in steps of $1 \mathrm{~mm}$, using an XYZ-axes rotary stage (HPS80-50X-M5, Sigma Koki Co., Tokyo, Japan), which was controlled using LabVIEW (National Instruments Co., TX, USA). Data acquisition took about 12 $\mathrm{min}$. Each projection data set was then transformed into 64 bins by linear interpolation. Both the inhomogeneous sensitivity of the receiving coil and feed through interference were corrected using the method described in [21]. Transverse images were reconstructed from the projection data using the ML-EM algorithm over 15 iterations, in which the initial concentration of MNPs was assumed to be uniform [17] [18]. In this study, we defined an MPI value as the pixel value of the transverse image reconstructed from the third-harmonic signals.

\subsection{System for Magnetic Hyperthermia}

The details of our system for magnetic hyperthermia are described in our previous paper [22]. In brief, an alternating magnetic field was generated with use of an external coil comprising 19 -turned loops $(6.5 \mathrm{~cm}$ in diameter and $10 \mathrm{~cm}$ in length) of copper pipe ( $5 \mathrm{~mm}$ in diameter) and was cooled by water to ensure constant temperature and impedance. The coil was connected to a power supply (T162-5723BHE, Thamway Co., Shizuoka, Japan) through an impedance tuner (T020-5723AHE, Thamway Co., Shizuoka, Japan). This system induced an alternating magnetic field with a maximum peak amplitude of $3.7 \mathrm{kA} / \mathrm{m}$ at an output power of $500 \mathrm{~W}$. The peak amplitude of the alternating magnetic field can be controlled by changing the output of the power supply. In this study, the frequency and peak amplitude of the alternating magnetic field were taken as $600 \mathrm{kHz}$ and $3.1 \mathrm{kA} / \mathrm{m}$, respectively.

\subsection{Magnetic Nanoparticles}

Resovist $^{\circledR}$ was used as the source of MNPs and was purchased from Fuji Film RI Pharma Co. (Tokyo, Japan). Resovist ${ }^{\circledR}$ consists of iron oxide (maghemite, $\gamma-\mathrm{Fe}_{2} \mathrm{O}_{3}$ ) nanoparticles coated with carboxydextran [22]-[24]. It is an organ-specific contrast agent for magnetic resonance imaging, used especially for the detection and characterization of small focal liver lesions.

\subsection{Phantom Experiments}

In phantom studies, samples with various iron concentrations of Resovist ${ }^{\circledR}(0,50,100,125,250$, and $500 \mathrm{mM})$ were prepared by putting Resovist ${ }^{\circledR}$ into a cylindrical polyethylene tube $6 \mathrm{~mm}$ in diameter and $5 \mathrm{~mm}$ in length $(100 \mu \mathrm{L})$ and were imaged using our MPI scanner [17]-[20]. These samples were also heated using our apparatus for magnetic hyperthermia [22] at $600 \mathrm{kHz}$ and $3.1 \mathrm{kA} / \mathrm{m}$, and the time course of the temperature was measured using an infrared thermometer (FLIR E4, FLIR Systems Inc., OR, USA) every $30 \mathrm{~s}$ during the first 2 min and every 1 min during the next 8 min after the beginning of magnetic hyperthermia. It should be noted that because the molar mass of iron $(\mathrm{Fe})$ is $55.8 \mathrm{~g} / \mathrm{mol}$, iron concentrations of $50,100,125,250$, and $500 \mathrm{mM}$ correspond to $2.8,5.6,7.0,14.0$, and $27.9 \mathrm{mg} \mathrm{Fe} / \mathrm{mL}$, respectively.

In general, the specific loss power (SLP) is used for characterizing the heating property of MNPs. The SLP in $\mathrm{W} / \mathrm{g}$ was calculated by the formula [25]

$$
S L P=\frac{C_{w} m_{w}+C_{\mathrm{Fe}} m_{\mathrm{Fe}}}{m_{\mathrm{Fe}}}\left(\frac{\Delta T}{\Delta t}\right)_{0},
$$

where $(\Delta T / \Delta t)_{0}$ is the initial slope of the time-dependent temperature rise, $C_{\mathrm{w}}$ is the specific heat capacity of water, $C_{\mathrm{Fe}}$ is the specific heat capacity of iron, $m_{\mathrm{w}}$ is the mass of water in the fluid per unit volume of fluid, and $m_{\mathrm{Fe}}$ 
is the mass of iron in the fluid per unit volume of fluid. The time-dependent temperature rise $(\Delta T(t))$ was fitted by use of the phenomenological Box-Lucas equation given by [26]

$$
\Delta T(t)=T(t)-T(0)=A\left(1-\mathrm{e}^{-B t}\right),
$$

where $T(t)$ and $T(0)$ are the temperatures at time $t$ and 0 , respectively, and $A$ and $B$ are constants. This equation is often used to describe the heating of MNPs using an alternating magnetic field [25]. The product of the fitting parameters, $A \times B$, is equivalent to $(\Delta T / \Delta t)_{0}$, and the SLP value was calculated from $(\Delta T / \Delta t)_{0}$ using Equation (1). In this study, we assumed $C_{\mathrm{w}}$ and $C_{\mathrm{Fe}}$ as $4.185 \mathrm{~J} / \mathrm{g} / \mathrm{K}$ and $0.746 \mathrm{~J} / \mathrm{g} / \mathrm{K}$, respectively. We also assumed $m_{\mathrm{w}}$ as 1 $\mathrm{g} / \mathrm{mL}$ and $m_{\mathrm{Fe}}$ as $5.58 \mathrm{mg} / \mathrm{mL}$ for $100 \mathrm{mM} \mathrm{Fe}$ of Resovist ${ }^{\mathbb{B}}$.

To investigate the relationship between the MPI value and the loss power per unit volume of solution, we introduced a new parameter, which we call the "volume-specific loss power (vSLP)" and is defined by

$$
\nu S L P=S L P \times m_{\mathrm{Fe}} .
$$

This parameter has a unit of $\mathrm{W} / \mathrm{mL}$.

\subsection{Animal Experiments}

Seven-week-old male BALB/c mice were purchased from Charles River Laboratories Japan, Inc. (Yokohama, Japan), and were habituated to the rearing environment for one week before the experiment. The animals had free access to food and water, and were kept under standard laboratory conditions of $22^{\circ} \mathrm{C}-23^{\circ} \mathrm{C}$ room temperatures, around $50 \%$ humidity, and a 12:12 hour light/dark cycle. After one-week habituation, Colon- 26 cells $(1 \times$ $10^{6}$ cells) were implanted subcutaneously into the back of a mouse under anesthesia by pentobarbital sodium (Somnopentyl, Kyoritsu Seiyaku Co., Tokyo, Japan) $(0.012 \mathrm{~mL} / \mathrm{g}$ body weight). Tumor volumes in all mice were measured every day. When the tumor volume reached approximately $100 \mathrm{~mm}^{3}$, mice were divided into untreated and treated groups. The tumors in the mice of the untreated group $(n=10)$ were injected with saline as a control, while those in the mice of the treated group were injected with Resovist ${ }^{\mathbb{B}}$ at an iron concentration of 250 $\mathrm{mM}$ (half-dose group) $(\mathrm{n}=10)$ or $500 \mathrm{mM}$ (full-dose group) $(\mathrm{n}=10)$. In this study, we injected saline or Resovist $^{\circledR}$ directly into the center of the tumor using a 26-gauge needle under anesthesia by pentobarbital sodium $(0.012 \mathrm{~mL} / \mathrm{g}$ body weight). The injected volume in the half-dose group was adjusted to be the same $(0.2 \mathrm{~mL})$ as that in the full-dose group by adding saline into Resovist ${ }^{\mathbb{R}}$ at an iron concentration of $500 \mathrm{mM}$.

Ten minutes after the injection of Resovist ${ }^{\circledR}$, MPI images were obtained in the same manner as in the phantom studies. In this study, one slice of the MPI image with the maximum signal intensity was obtained per mouse. After the MPI studies, X-ray CT images were obtained using a 4-row multi-slice CT scanner (Asteion, Toshiba Medical Systems Co., Tochigi, Japan) with a tube voltage of $120 \mathrm{kV}$ and a tube current of $210 \mathrm{~mA}$. The MPI image was co-registered with the X-ray CT image using parameters for magnification and rotation that were previously obtained using a phantom with 3 point sources with a diameter of $0.5 \mathrm{~mm}$ and filled with $100 \mathrm{mM} \mathrm{Fe}$ MNPs.

Thirty minutes after the injection of Resovist ${ }^{\circledR}$, magnetic hyperthermia treatment was performed by applying an alternating magnetic field at $600 \mathrm{kHz}$ and $3.1 \mathrm{kA} / \mathrm{m}$ for $20 \mathrm{~min}$. During magnetic hyperthermia treatment, the temperatures in the tumor and rectum were measured using two fluorescence-type optical-fiber thermometers (FL-2000, Anritsu Meter Co., Tokyo, Japan); one was placed in the tumor, and the other was inserted $1 \mathrm{~cm}$ inside the rectum.

Tumor volumes in all mice were measured with a caliper every day for 15 days. The tumor volumes $(V$ in $\mathrm{mm}^{3}$ ) were calculated as $V=(\pi / 6) \times L_{\mathrm{x}} \times L_{\mathrm{y}} \times L_{\mathrm{z}}$, where $L_{\mathrm{x}}, L_{\mathrm{y}}$, and $L_{\mathrm{z}}$ denote the vertical diameter, the horizontal diameter, and the height in mm, respectively. The relative tumor volume growth was calculated from $(V-$ $\left.V_{0}\right) / V_{0}$, where $V_{0}$ represents the tumor volume immediately before magnetic hyperthermia treatment. In this study, the relative tumor volume growth at 15 days after magnetic hyperthermia treatment was used as an index of the therapeutic effect of magnetic hyperthermia treatment.

All animal experiments described above were approved by the animal ethics committee at Osaka University School of Medicine.

\subsection{Statistical Analysis}

In this study, the MPI value was defined as the pixel value of the transverse image reconstructed from the 
third-harmonic signals, as previously described. In phantom experiments, we calculated the average MPI values within a circular region of interest (ROI) with a diameter of $6 \mathrm{~mm}$, drawn on the MPI images. In animal experiments, we calculated the average MPI values within the ROI drawn on the tumors by taking the threshold value for extracting the contour as $20 \%$ of the maximum MPI value in the ROI.

Unless specifically stated, the average MPI value, temperature rise, tumor volume, and relative tumor volume growth were expressed as the mean \pm standard error (SE). Differences in these parameters among groups were analyzed by one-way analysis of variance (ANOVA). Statistical significance was determined by Tukey's multiple comparison test. A $P$ value less than 0.05 was considered statistically significant.

\section{Results}

The results of phantom experiments are shown in Figures 1-5. Figure 1(a) shows the typical example of the MPI image of a phantom containing $100 \mathrm{mM} \mathrm{Fe} \mathrm{Resovist}{ }^{\circledR}$. Figure 1 (b) shows the thermal image of the phan-

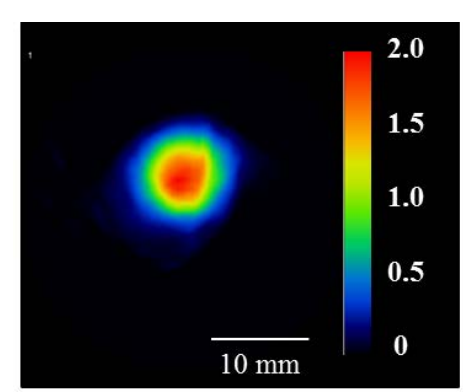

(a)

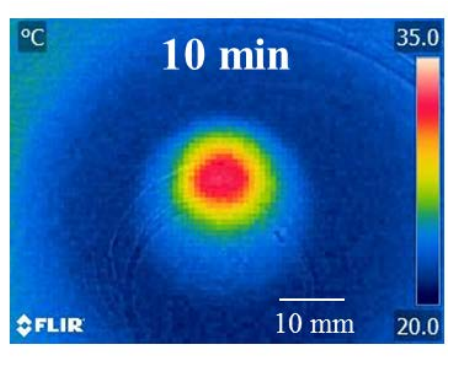

(b)

Figure 1. Results of phantom experiments. (a) Typical example of the image of a phantom (6 $\mathrm{mm}$ in diameter and $5 \mathrm{~mm}$ in length) filled with magnetic nanoparticles (Resovist ${ }^{\circledR}$ ), which was obtained by magnetic particle imaging (MPI). In this case, the volume and iron concentration of Resovist ${ }^{\mathbb{B}}$ were $100 \mu \mathrm{L}$ and $100 \mathrm{mM}$, respectively. Scale bar $=10$ $\mathrm{mm}$. (b) Thermal image of the phantom obtained by an infrared thermometer $10 \mathrm{~min}$ after the start of magnetic hyperthermia. Note that the lowest and highest levels for display were set at $20^{\circ} \mathrm{C}$ and $35^{\circ} \mathrm{C}$, respectively. Scale bar $=10 \mathrm{~mm}$.

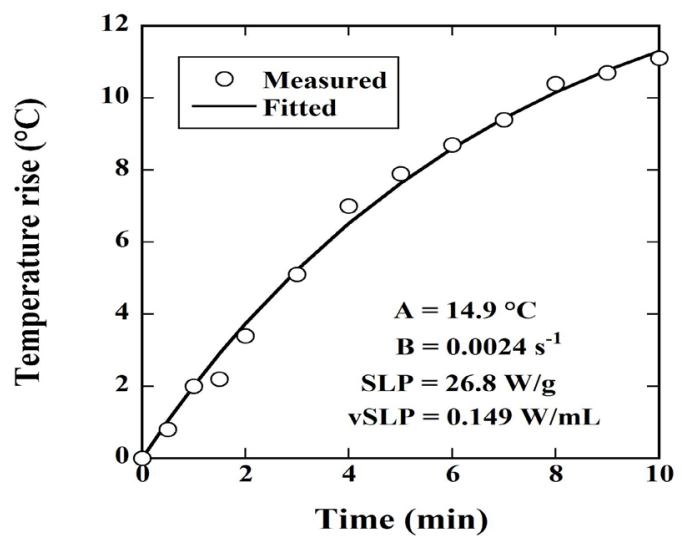

Figure 2. Results of phantom experiments. Typical example of the time course of the temperature rise in the phantom obtained from the thermal images (open circles). The solid line represents the fitted curve using the phenomenological Box-Lucas equation (Equation (2)), from which $A$ and $B$ were obtained for calculating the specific loss power (SLP) and volume-specific loss power (vSLP) using Equations (1) and (3), respectively. 


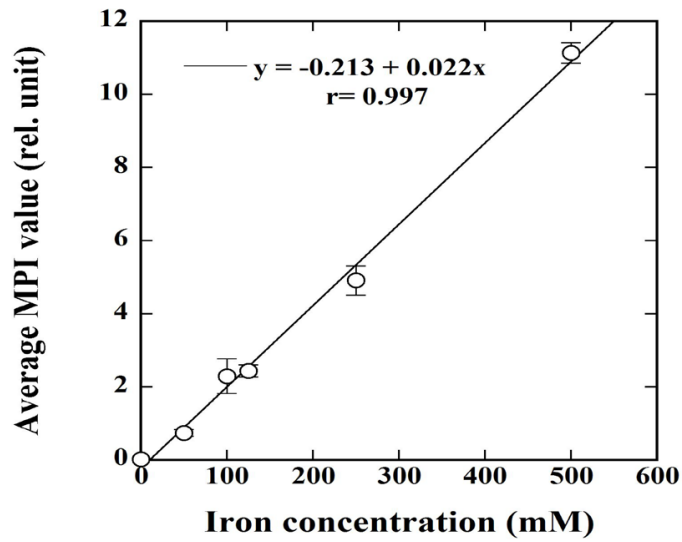

Figure 3. Results of phantom experiments. Correlation between the average MPI value and the iron concentration of Resovist ${ }^{\mathbb{B}}$. The MPI value was defined as the pixel value of the transverse image reconstructed from the third-harmonic signals, and the average MPI value was calculated by drawing a circular region of interest (ROI) with a diameter of $6 \mathrm{~mm}$ on the MPI image. Data are represented by mean \pm standard error $(\mathrm{SE})$.

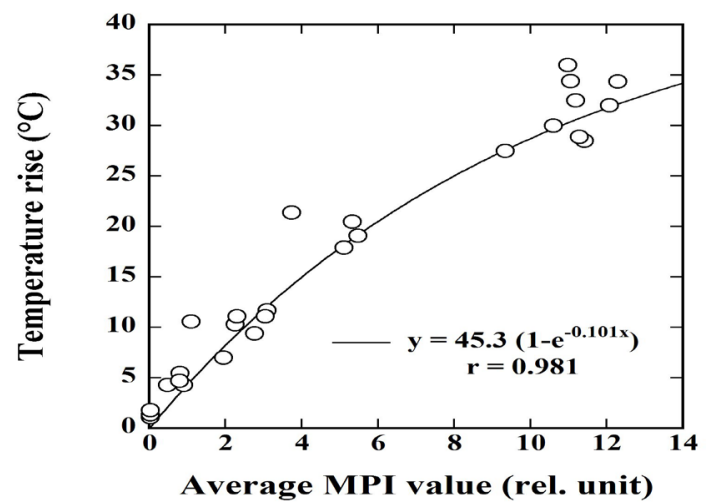

Figure 4. Results of phantom experiments. Correlation between the average MPI value and the temperature rise $10 \mathrm{~min}$ after the start of magnetic hyperthermia, measured by an infrared thermometer.

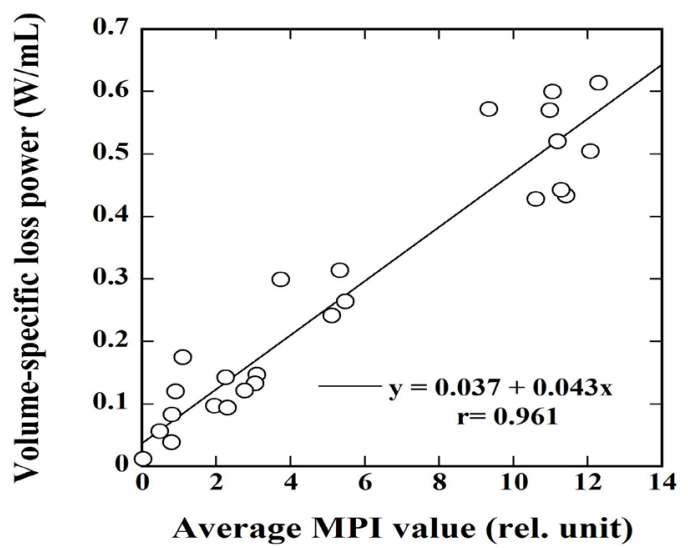

Figure 5. Results of phantom experiments. Correlation between the average MPI value and the vSLP value. The vSLP value was calculated from Equation (3). 
tom obtained by an infrared thermometer $10 \mathrm{~min}$ after the start of magnetic hyperthermia. Figure 2 shows the typical example of the time course of the temperature rise in the phantom obtained from the thermal images (open circles) and the fitted curve using the phenomenological Box-Lucas equation (Equation (2)) (solid line). In this case, the SLP and vSLP values were calculated to be $26.8 \mathrm{~W} / \mathrm{g}$ and $0.149 \mathrm{~W} / \mathrm{mL}$ using Equations (1) and (3), respectively.

Figure 3 shows the correlation between the average MPI value and the iron concentration of Resovist ${ }^{\circledR}$. As shown in Figure 3, there was an excellent correlation between them $(r=0.997)$. Figure 4 shows the correlation between the average MPI value and the temperature rise $10 \mathrm{~min}$ after the start of magnetic hyperthermia. As shown in Figure 4, when regression analysis was performed using the phenomenological Box-Lucas equation given by Equation (2), there was an excellent correlation between them $(r=0.981)$. There was no significant correlation between the average MPI value (x) and the SLP value calculated from Equation (1) (y) (y $=-0.530 \mathrm{x}+$ $24.1, r=0.364)$ (plot not shown), and the SLP value was almost constant regardless of the MPI value (20.9 \pm 6.4 $\mathrm{W} / \mathrm{g}$ (mean \pm standard deviation (SD))). Figure 5 shows the correlation between the average MPI value and the vSLP value calculated from Equation (3). There was also an excellent correlation between them $(r=0.961)$.

The results of in vivo animal experiments are shown in Figures 6-11. Figures 6(a)-(b) show typical examples

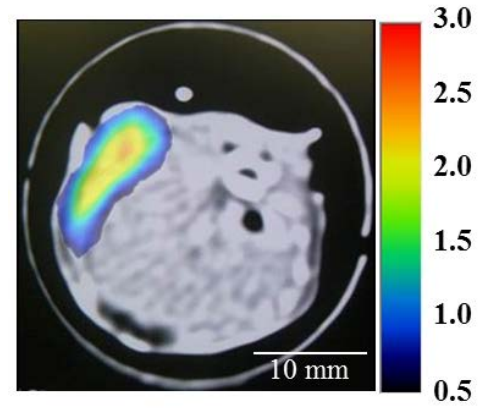

(a)

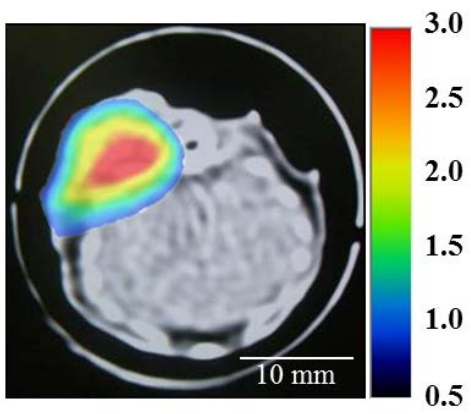

(b)

Figure 6. Results of animal experiments. (a) Typical example of the MPI image of a tumor-bearing mouse injected with Resovist $^{\circledR}$ at an iron concentration of $250 \mathrm{mM}$ and a volume of $0.2 \mathrm{~mL}$, which was superimposed on the X-ray CT image. Scale bar $=10 \mathrm{~mm}$. (b) Typical example of the MPI image of a tumor-bearing mouse injected with Resovist ${ }^{\circledR}$ at an iron concentration of $500 \mathrm{mM}$ and a volume of $0.2 \mathrm{~mL}$, which was superimposed on the X-ray CT image. Scale bar $=10 \mathrm{~mm}$.

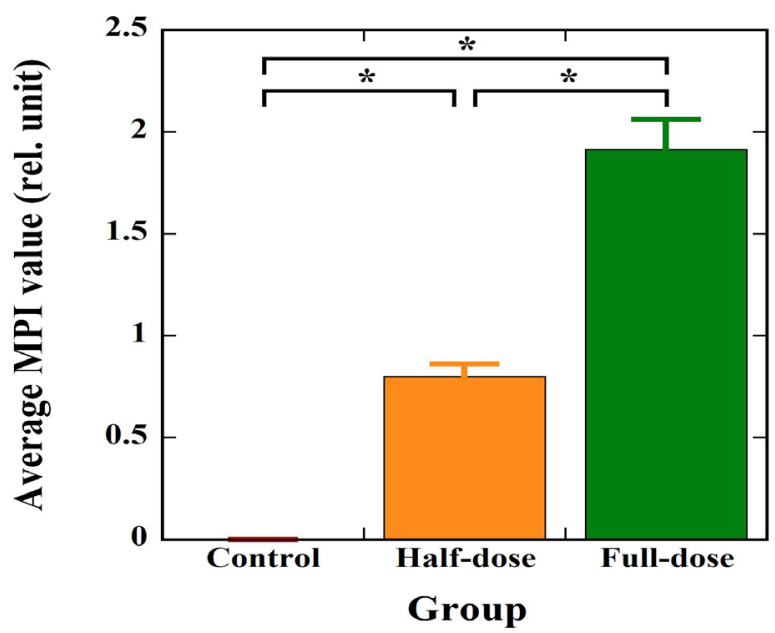

Figure 7. Results of animal experiments. Comparison of the average MPI values among the control, half-dose, and full-dose groups. The tumors in the mice of the control group $(\mathrm{n}=10)$ were injected with saline, while those of the half-dose $(\mathrm{n}=10)$ and full-dose groups $(\mathrm{n}=10)$ were injected with Resovist ${ }^{\mathbb{R}}$ at iron concentrations of $250 \mathrm{mM}$ and $500 \mathrm{mM}$, respectively. The average MPI value was calculated by drawing an ROI on the tumor by taking the threshold value for extracting the contour as $20 \%$ of the maximum MPI value in the ROI. Note that the average MPI value in the control group was taken as zero. Bar and error bar represent mean and SE, respectively. ${ }^{*} P<0.05$. 


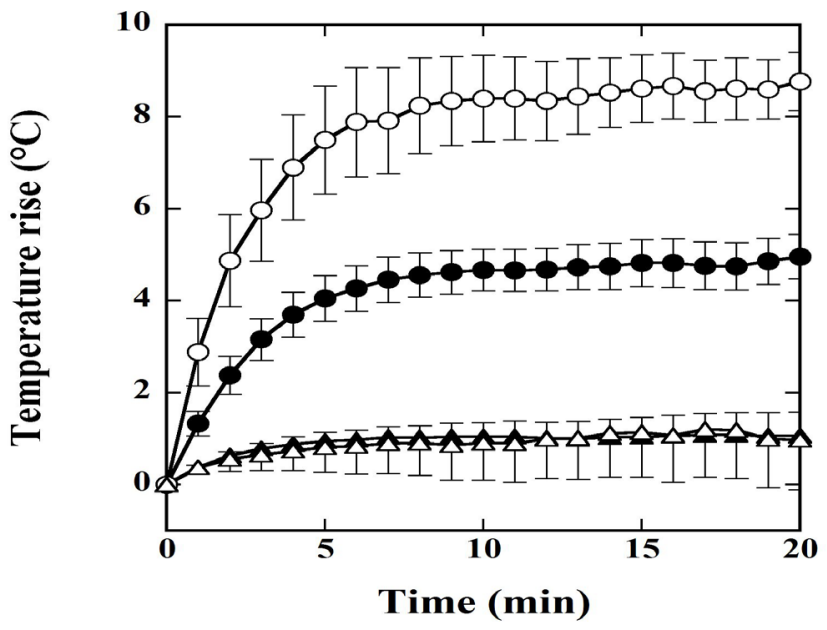

Figure 8. Results of animal experiments. Time courses of the temperature rise after the start of magnetic hyperthermia in the tumor and rectum in the half-dose and full-dose groups. The closed circles and closed triangles show the time courses in the tumor and rectum in the half-dose group, respectively, while the open circles and open triangles show those in the full-dose group. Data are represented by mean $\pm \mathrm{SE}$.

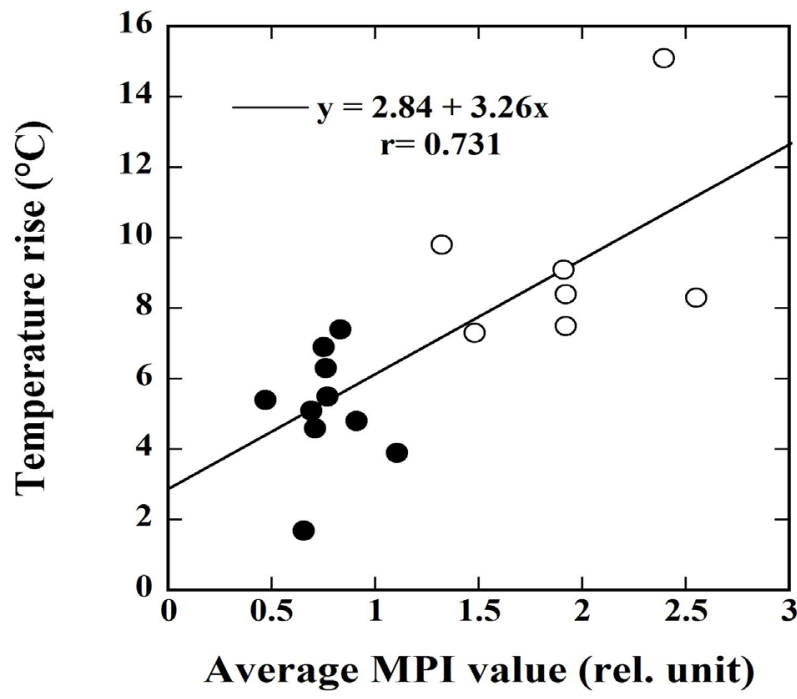

Figure 9. Results of animal experiments. Correlation between the average MPI value and the temperature rise in the tumor $20 \mathrm{~min}$ after the start of magnetic hyperthermia in the half-dose (closed circles) and full-dose groups (open circles). Note that data of three mice in the full-dose group were removed because the temperature in the tumor in these mice was not successfully measured.

of the MPI images of tumor-bearing mice injected with $250 \mathrm{mM} \mathrm{Fe}$ (half-dose group) and $500 \mathrm{mM}$ Fe Resovist ${ }^{\circledR}$ (full-dose group), respectively, which were superimposed on the X-ray CT images. The distribution of MNPs obtained by MPI correlated well with that of the tumor in the X-ray CT image in both cases. The MPI values in Figures 6(a)-(b) were $1.10 \pm 0.60$ (mean $\pm \mathrm{SD}$ ) and $1.64 \pm 0.83$ (mean $\pm \mathrm{SD}$ ), respectively.

Figure 7 shows the comparison of the average MPI value among groups. It should be noted that the average MPI value in the control group was taken as zero. As shown in Figure 7, there were significant differences among groups. 


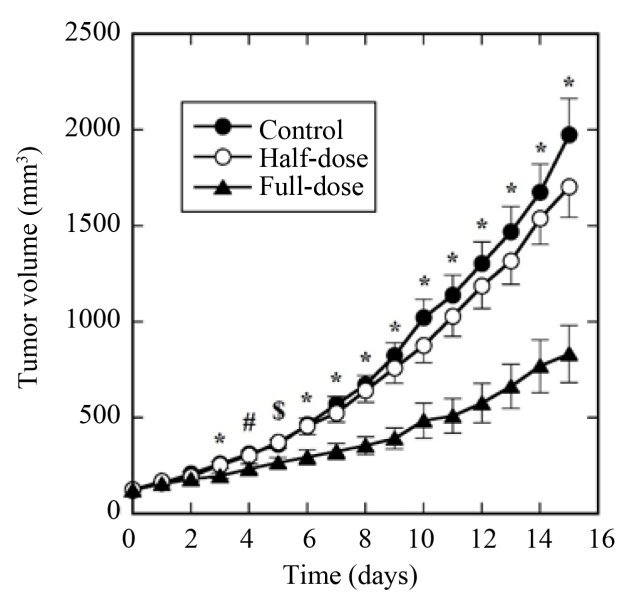

(a)

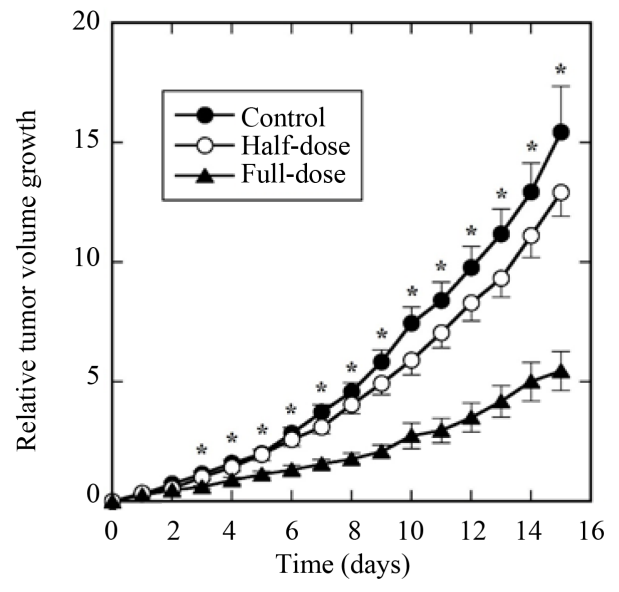

(b)

Figure 10. Results of animal experiments. (a) Time courses of the tumor volume after magnetic hyperthermia treatment in the control (closed circles), half-dose (open circles), and full-dose groups (closed triangles). Data are represented by mean \pm SE. ${ }^{\#} P<0.05$ between the control and full-dose groups, ${ }^{\$} P<0.05$ between the half-dose and full-dose groups, and ${ }^{*} P<0.05$ between the control and full-dose groups and between the half-dose and full-dose groups. (b) Time courses of the relative tumor volume growth after magnetic hyperthermia treatment in the control (closed circles), half-dose (open circles), and full-dose groups (closed triangles). The relative tumor volume growth was calculated from $\left(V-V_{0}\right) / V_{0}$, where $V_{0}$ and $V$ represent the tumor volumes immediately before and after magnetic hyperthermia treatment, respectively. Data are represented by mean $\pm \mathrm{SE}$. ${ }^{*} P<$ 0.05 between the control and full-dose groups and between the half-dose and full-dose groups.

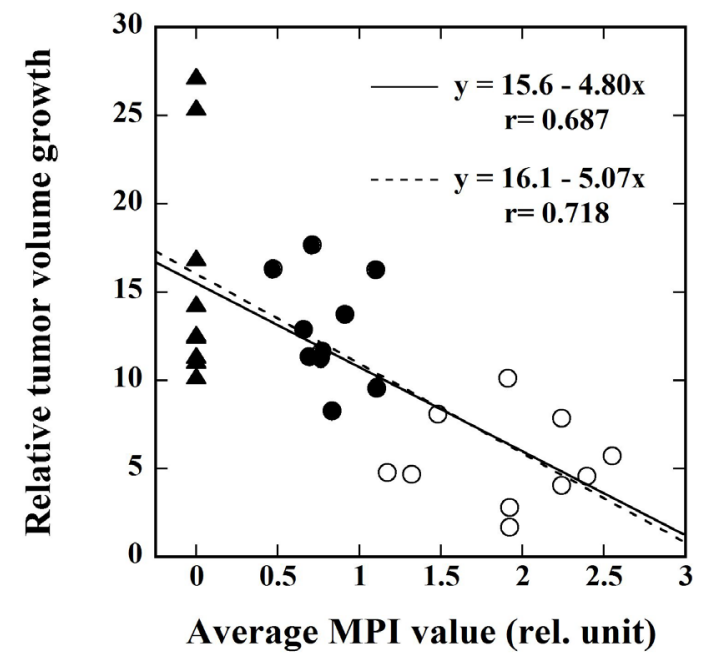

Figure 11. Results of animal experiments. Correlation between the average MPI value and the relative tumor volume growth at 15 days after magnetic hyperthermia treatment in the control (closed triangles), half-dose (closed circles), and full-dose groups (open circles). Note that the average MPI values in the control group were taken as zero. The solid line represents the regression line when the data in all groups were used, while the dashed line represents the case when only the data in the treated group were used.

Figure 8 shows the time courses of the temperature rise after the start of magnetic hyperthermia in the tumor and rectum in the half-dose and full-dose groups. The closed circles and closed triangles show the temperature rises in the tumor and rectum in the half-dose group, respectively, while the open circles and open triangles show those in the full-dose group. Although the temperature in the rectum did not rise significantly in both the half-dose and full-dose groups, the temperature rise in the tumor in the full-dose group was higher than that in 
the half-dose group by a factor of approximately 2 .

Figure 9 shows the relationship between the average MPI value and the temperature rise in the tumor $20 \mathrm{~min}$ after the start of magnetic hyperthermia in the half-dose (closed circles) and full-dose groups (open circles). The average MPI value had a significant correlation with the temperature rise in the tumor $(r=0.731)$. It should be noted that data of three mice in the full-dose group were removed because the temperature in the tumor in these mice was not successfully measured.

Figure 10(a) shows the time courses of the tumor volume after magnetic hyperthermia treatment in the control (closed circles), half-dose (open circles), and full-dose groups (closed triangles), while Figure 10(b) shows the time courses of the relative tumor volume growth in the control (closed circles), half-dose (open circles), and full-dose groups (closed triangles). As shown in Figure 10, significant differences in both the tumor volume and relative tumor volume growth between the control and full-dose groups and between the half-dose and full-dose groups were observed after 3 days of magnetic hyperthermia treatment.

Figure 11 shows the correlation between the average MPI value and the relative tumor volume growth at 15 days after magnetic hyperthermia treatment when the data in all groups were used (solid regression line) or only the data in the treated group, i.e., half-dose (closed circles) and full-dose groups (open circles) were used (dashed regression line). Note that the average MPI values in the control group (closed triangles) were taken as zero. As shown in Figure 11, there was a significant negative correlation between them $(r=0.687)$ for the data in all groups. The correlation coefficient between them was slightly increased for the data in the treated group $(\mathrm{r}=0.718)$.

\section{Discussion}

In this study, we investigated the feasibility of MPI for predicting the therapeutic effect of magnetic hyperthermia treatment using phantom and in vivo small animal experiments. Our preliminary results (Figures 1-11) suggest that MPI is feasible and useful for predicting the therapeutic effect of magnetic hyperthermia treatment. To the best of our knowledge, this is the first report on the application of MPI to magnetic hyperthermia.

Our phantom experiments (Figures 1-5) demonstrated that the average MPI value has excellent linear correlations with the iron concentration and VSLP of MNPs, indicating that MPI can not only visualize the spatial distribution of MNPs but also quantify the concentration of MNPs and predict the temperature rise induced by magnetic hyperthermia. These aspects appear to be very important for the effective and safe treatment planning of magnetic hyperthermia treatment. As shown in Figure 4, however, a large scatter of the temperature rise was observed. As previously described, the temperature rise was measured using an infrared thermometer instead of an optical-fiber thermometer to compare the thermal image with the MPI image. Although the infrared thermometer allows us to obtain the image of the surface temperature (not internal temperature), the accuracy of this thermometer is affected by several factors such as the condition of the target surface, the distance between the camera and the target surface, and the angle of the camera axis with respect to the target surface norm [27]. In addition, the temperature of the phantom itself is also affected by several factors such as conduction, i.e., the heat transfer between the medium in the phantom and adjoining materials and convection, i.e., the heat transfer between the phantom and its surroundings [28]. These factors appear to have caused a large scatter of the temperature rise observed in Figure 4.

As shown in Figure 5, a large scatter was also observed in the vSLP value. As previously described, the vSLP value was calculated from $(\Delta T / \Delta t)_{0}$, which was obtained by fitting the time course of the temperature rise by use of the phenomenological Box-Lucas equation [26]. This equation is based on the assumption that the heat loss is linearly dependent on the difference in temperature between the sample and its surroundings [28]. If we take into account all possible losses, the relation between the temperature and heat loss becomes nonlinear. Thus, this linear-loss assumption in calculating $(\Delta T / \Delta t)_{0}$ may also be one of the factors causing a large scatter of the vSLP value observed in Figure 5.

As shown in Figure 6, our MPI scanner could visualize the spatial distribution of MNPs in the tumor as a positive contrast. This excellent visibility of MPI appears to be useful for not only the diagnosis but also the treatment planning. Our animal experiments (Figure 9) also demonstrated that there was a significant positive correlation between the average MPI value and the temperature rise in the tumor, suggesting that MPI can also estimate the temperature rise induced by magnetic hyperthermia in vivo. As shown in Figure 9, however, a large scatter was observed in the temperature rise in the tumor. This appears to be mainly due to the fact that the dis- 
tribution of MNPs in the tumor is not homogeneous as shown in Figure 6 and thus the temperature rise measured by an optical-fiber thermometer is largely dependent on the position of the probe inserted into the tumor. Furthermore, a significant negative correlation was observed between the average MPI value and the relative tumor volume growth at 15 days after magnetic hyperthermia treatment (Figure 11), suggesting that we can predict the therapeutic effect of magnetic hyperthermia treatment using the pixel value of the MPI image.

When we consider application of MPI, it is important to know whether the temperature affects the MPI signal. To investigate the effect of the temperature on the MPI signal, we measured the MPI signal at three different temperatures $\left(30^{\circ} \mathrm{C}, 40^{\circ} \mathrm{C}\right.$, and $\left.50^{\circ} \mathrm{C}\right)$ using a phantom described previously at an iron concentration of $100 \mathrm{mM}$. The MPI signal normalized by that at $30^{\circ} \mathrm{C}$ was $0.97 \pm 0.01($ mean $\pm \mathrm{SD})$ and $0.95 \pm 0.01$ (mean $\pm \mathrm{SD}$ ) at $40^{\circ} \mathrm{C}$ and $50{ }^{\circ} \mathrm{C}$, respectively, implying that the MPI signal decreased by approximately $0.3 \% /{ }^{\circ} \mathrm{C}$ with increasing temperature. When we measured the temperature of samples before and after the MPI studies using an infrared thermometer, the temperature of samples did not change significantly. Thus, the temperature-dependent change in the MPI signal appears to be negligible during the data acquisition of MPI.

The degree to which magnetic hyperthermia can be applied to cancer therapy depends on the ability to deliver MNPs systematically to tumor cells in sufficient concentrations. The delivery of adequate numbers of MNPs to the tumor site via systemic administration remains challenging. If MNPs were adsorbed only to tumor cells, the MNPs could be administered intravenously. This feature would be of great advantage in terms of the quality of life of patients. However, because the administered MNPs migrate passively to a mononuclear phagocyte system such as the Kupffer cells of the liver and spleen, the passive targeting of MNPs for cancer is a very important issue for the establishment of cancer therapy with use of magnetic hyperthermia. With regard to the delivery of MNPs, magnetoliposomes may be a promising tool for passive targeting. Shinkai et al. [29] developed magnetite cationic liposomes (MCLs) with improved absorption and accumulation properties within tumors. Administration of the MCLs, however, is limited to direct injection into the tumor tissue [12]. The conjugation of antibodies to MNPs is a possible approach to achieving the passive targeting of MNPs for cancer. Le et al. [30] and Shinkai et al. [31] have developed MNPs conjugated to the Fab' fragments of anti-human MN antigen-specific antibody. However, because the target concentration is very low in antibody targeting of tumors [32], this approach cannot yet be translated successfully from research to the clinical stage. Gilchrist et al. [4] tried selective hyperthermia by direct injection of a suspension of MNPs into draining lymph nodes from colon cancer during surgical operation and successfully heated them. This technology is the first heating technique by use of MNPs to have entered clinical trials. Moroz et al. [33] assessed the use of ferromagnetic embolization hyperthermia, which consists of arterially embolizing liver tumors with ferromagnetic particles in a rabbit liver tumor model, and they reported that hepatic arterial infusion of lipiodol-containing ferromagnetic particles can result in excellent targeting of liver tumors with hyperthermia by subsequent application of an alternating magnetic field. In this study, we injected Resovist ${ }^{\circledR}$ into the tumor tissue directly to achieve selective hyperthermia via direct injection of MNPs or transarterial embolization, because an intravenous-injection approach to achieving the passive targeting of MNPs for cancer is not yet available in the clinical setting, as mentioned above. In addition, magnetic hyperthermia treatment was performed only once for each mouse in the treated group and the observation period of tumor growth was relatively short (15 days), to focus on the investigation of the feasibility of MPI for predicting the therapeutic effect rather than the usefulness of magnetic hyperthermia itself.

Although all the mice in either the half-dose or the full-dose groups were injected with the same dose of Resovist $^{\circledR}$, some variation of the average MPI value was observed (Figure 7, Figure 9, and Figure 11). When we injected Resovist ${ }^{\circledR}$ into the tumor tissue directly, dilution and/or loss of Resovist ${ }^{\circledR}$ would occur due to washout via blood perfusion and/or diffusion in the targeted tissue. This appears to be the main reason for the above finding (Figure 7, Figure 9, and Figure 11). In this study, we performed MPI studies before applying magnetic hyperthermia. The change in the distribution and quantity of MNPs within the tumor after magnetic hyperthermia and its linkage with the therapeutic effect are of great interest, especially when magnetic hyperthermia treatment is performed repeatedly. If we performed MPI studies before and after magnetic hyperthermia, we could assess the change in the distribution and quantity of MNPs using the MPI images acquired before and after magnetic hyperthermia. These studies are currently in progress.

As previously described, the threshold value for drawing the ROI for calculating the average MPI value in the tumor was taken as $20 \%$ of the maximum MPI value in the ROI. This threshold value was determined visually from inspection of the MPI images with various threshold values, which were superimposed on the X-ray CT image. Although this threshold value appears to be appropriate in our experience, further studies on the optimi- 
zation of the threshold value may be necessary.

A limitation of this study is that the MPI value was obtained from a single slice of the MPI image with the maximum signal intensity. For more detailed analysis, three-dimensional (multi-slice) data will be necessary. If three-dimensional data were obtained, the usefulness and accuracy of MPI for predicting the therapeutic effect of magnetic hyperthermia treatment would increase. These studies are also in progress.

One of the challenging problems in magnetic hyperthermia treatment is to estimate the influence of blood flow on the temperature rise in the tissue. In real conditions, the heat transferred by blood flow should also be considered in the analysis of temperature rise during magnetic hyperthermia treatment. The relationship between temperature response and blood perfusion has been studied by several investigators [34] [35]. Bioheat transfer analysis often needs to deal simultaneously with transient and spatial heating inside the tissue. The Pennes bioheat transfer equation [36], which describes the exchange magnitude of the heat transfer between tissue and blood, has been used widely for solving the temperature distribution for thermal therapy [37]. Information on the spatial distribution and quantity of MNPs is necessary as initial and boundary conditions to solve the Pennes bioheat transfer equation [36] accurately. Thus, we will perform a more detailed analysis by solving the Pennes bioheat transfer equation [36] based on the MPI images representing the spatial distribution and quantity of MNPs. We believe that MPI will be useful for providing the initial and boundary conditions for solving the Pennes bioheat transfer equation [36].

As previously described, the frequency of the drive magnetic field used in MPI was $400 \mathrm{~Hz}$ in this study, which is too low to be used for magnetic hyperthermia. If we switch this frequency to one appropriate for magnetic hyperthermia (600 kHz in this study) after imaging and diagnosing the tumor using MPI, we may realize so-called "theranostics", in which diagnostics and therapy are integrated into a single platform; this concept has recently gained increased interest [38]. Recently, we demonstrated that it is possible to control the temperature rise in magnetic hyperthermia with use of an external static magnetic field [39]. If we use the selection magnetic field in MPI as the static magnetic field for controlling the temperature rise, it may be possible to realize local control of the temperature rise in magnetic hyperthermia to prevent damage to the surrounding healthy tissue, resulting in a reduction of the side effects induced by magnetic hyperthermia [39].

In this study, the frequency $(f)$ and peak amplitude $(H)$ of an alternating magnetic field for magnetic hyperthermia were taken as $600 \mathrm{kHz}$ and $3.1 \mathrm{kA} / \mathrm{m}$, respectively. Atsumi et al. [40] used $600 \mathrm{kHz}$ for $f$ in consideration of the safety and the capacity of their power supply. Thus, we also selected the above value for $f$ in consideration of safety, the capacity of our power supply, and the heating efficiency [22]. When we consider practical application of the present method, however, $f$ and $H$ must be rightfully chosen, because high $f$ and $H$ can lead to unwanted damage to the surrounding healthy tissue via eddy currents [41]. Experimentally, Brezovich et al. [42] found for a loop diameter of $30 \mathrm{~cm}$ that test persons were able to withstand the treatment for more than one hour without major discomfort if $f \cdot H<4.85 \times 10^{8} \mathrm{Am}^{-1} \cdot \mathrm{s}^{-1}$. Hergt et al. [43] assumed a weaker criterion of $f \cdot H<5 \times$ $10^{9} \mathrm{Am}^{-1} \cdot \mathrm{s}^{-1}$ for a smaller diameter of exposed body region and with dependence on the seriousness of the illness. Although the $f$ and $H$ values adopted in this study satisfy Hergt's criterion [43], the use of a lower $f$ may be preferable for clinical application.

Another imaging method to detect MNPs is magnetic resonance imaging (MRI). When we attempted to image MNPs using MRI with a conventional transverse relaxation time $\left(T_{2}^{*}\right)$-weighted imaging sequence, it was almost impossible due to large susceptibility-induced MR signal loss and image distortions in the regions near the MNPs. Recently, however, MRI pulse sequences capable of preserving the signal from spins with ultrashort $T_{2}^{*}$, such as ultrashort echo time (UTE) [44] and sweep imaging with Fourier transformation (SWIFT) sequences [45], have been developed. With these pulse sequences, MNPs can be detected and quantified based on the shortening of the longitudinal relaxation time of water $\left(T_{1}\right)$ [45]. Zhang et al. [46] reported that the reciprocal of $T_{1}$ measured using the SWIFT sequence combined with the Look-Locker method has a linear relationship with MNPs concentration up to $53.6 \mathrm{mM}$ of iron. Although their method also appears to be promising and useful for planning effective magnetic hyperthermia treatment, significant research and technology development remain to be done before establishing the effectiveness of their method when translated to animal and/or human studies [47].

\section{Conclusion}

Our preliminary results suggest that MPI can be useful for predicting the therapeutic effect of magnetic hyper- 
thermia treatment and will provide a new imaging strategy for establishing reliable and safe magnetic hyperthermia treatment in the clinical setting.

\section{Acknowledgements}

This work was supported by a Grant-in-Aid for Scientific Research (B) (Grant Number: 25282131) from the Japan Society for the Promotion of Science (JSPS).

\section{References}

[1] Abe, M., Hiraoka, M., Takahashi, M., Egawa, S., Matsuda, C., Onoyama, Y., Morita, K., Kakehi, M. and Sugahara, T. (1986) Multi-Institutional Studies on Hyperthermia Using an 8-MHz Radiofrequency Capacitive Heating Device (Thermotron RF-8) in Combination with Radiation for Cancer Therapy. Cancer, 58, 1589-1595. http://dx.doi.org/10.1002/1097-0142(19861015)58:8<1589::AID-CNCR2820580802>3.0.CO;2-B

[2] Oura, S., Tamaki, T., Hirai, I., Yoshimasu, T., Ohta, F., Nakamura, R. and Okamura, Y. (2007) Radiofrequency Ablation Therapy in Patients with Breast Cancers Two Centimeters or Less in Size. Breast Cancer, 14, 48-54. http://dx.doi.org/10.2325/jbcs. 14.48

[3] Seip, R. and Ebbini, E.S. (1995) Noninvasive Estimation of Tissue Temperature Response to Heating Fields Using Diagnostic Ultrasound. IEEE Transactions on Biomedical Engineering, 42, 828-839. http://dx.doi.org/10.1109/10.398644

[4] Gilchrist, R.K., Medal, R., Shorey, W.D., Hanselman, R.C., Parrott, J.C. and Taylor, C.B. (1957) Selective Inductive Heating of Lymph Nodes. Annals of Surgery, 146, 596-606. http://dx.doi.org/10.1097/00000658-195710000-00007

[5] Jordan, A., Scholz, R., Maier-Hauff, K., Johannsen, M., Wust, P., Nodobny, J., Schirra, H., Schmidt, H., Deger, S. and Leoning, S. (2001) Presentation of a New Magnetic Field Therapy System for the Treatment of Human Solid Tumors with Magnetic Fluid Hyperthermia. Journal of Magnetism and Magnetic Materials, 225, 118-126. http://dx.doi.org/10.1016/S0304-8853(00)01239-7

[6] Kozissnik, B., Bohorquez, A.C., Dobson, J. and Rinaldi, C. (2013) Magnetic Fluid Hyperthermia: Advances, Challenges, and Opportunity. International Journal of Hyperthermia, 29, 706-714. http://dx.doi.org/10.3109/02656736.2013.837200

[7] Hilger, I. (2013) In Vivo Applications of Magnetic Nanoparticle Hyperthermia. International Journal of Hyperthermia, 29, 828-834. http://dx.doi.org/10.3109/02656736.2013.832815

[8] Rosensweig, R.E. (2002) Heating Magnetic Fluid with Alternating Magnetic Field. Journal of Magnetism and Magnetic Materials, 252, 370-374. http://dx.doi.org/10.1016/S0304-8853(02)00706-0

[9] Neuberger, T., Schopf, B., Hofmann, H., Hofmann, M. and von Rechenberg, B. (2005) Superparamagnetic Nanoparticles for Biomedical Applications: Possibilities and Limitations of a New Drug Delivery System. Journal of Magnetism and Magnetic Materials, 293, 483-496. http://dx.doi.org/10.1016/j.jmmm.2005.01.064

[10] Grüttner, C., Müller, K., Teller, J. and Westphal, F. (2013) Synthesis and Functionalization of Magnetic Nanoparticles for Hyperthermia Applications. International Journal of Hyperthermia, 29, 777-789. http://dx.doi.org/10.3109/02656736.2013.835876

[11] Ito, A., Shinkai, M., Honda, H. and Kobayashi, T. (2005) Medical Applications of Functionalized Magnetic Nanoparticles. Journal of Bioscience and Bioengineering, 100, 1-11. http://dx.doi.org/10.1263/jbb.100.1

[12] Balivada, S., Rachakatla, R.S., Wang, H., Samarakoon, T.N., Dani, R.K., Pyle, M., Kroh, F.O., Walker, B., Leaym, X., Koper, O.B., Tamura, M., Chikan, V., Bossmann, S.H. and Troyer, D.L. (2010) A/C Magnetic Hyperthermia of Melanoma Mediated by Iron(0)/iron Oxide Core/shell Magnetic Nanoparticles: A Mouse Study. BMC Cancer, 10, 119-127. http://dx.doi.org/10.1186/1471-2407-10-119

[13] Johannsen, M., Gneueckow, U., Thiesen, B., Taymoorian, K., Cho, C.H., Waldofner, N., Scholz, R., Jordan, A., Loening, S.A. and Wust, P. (2007) Thermotherapy of Prostate Cancer Using Magnetic Nanoparticles: Feasibility, Imaging, and Three-Dimensional Temperature Distribution. European Urology, 52, 1653-1662. http://dx.doi.org/10.1016/j.eururo.2006.11.023

[14] LeBrun, A., Manuchehrabadi, N., Attaluri, A., Wang, F., Ma, R. and Zhu, L. (2013) MicroCT Image-Generated Tumour Geometry and SAR Distribution for Tumour Temperature Elevation Simulations in Magnetic Nanoparticle Hyperthermia. International Journal of Hyperthermia, 29, 730-738. http://dx.doi.org/10.3109/02656736.2013.836757

[15] Gleich, B. and Weizenecker, J. (2005) Tomographic Imaging Using the Nonlinear Response of Magnetic Particles. Nature, 435, 1214-1217. http://dx.doi.org/10.1038/nature03808

[16] Goodwill, P.W., Konkle, J.J., Zheng, B., Saritas, E.U. and Conolly, S.M. (2012) Projection X-Space Magnetic Particle 
Imaging. IEEE Transactions on Medical Imaging, 31, 1076-1085. http://dx.doi.org/10.1109/TMI.2012.2185247

[17] Murase, K., Hiratsuka, S., Song, R. and Takeuchi, Y. (2014) Development of a System for Magnetic Particle Imaging using Neodymium Magnets and Gradiometer. Japanese Journal of Applied Physics, 53, Article ID: 067001. http://dx.doi.org/10.7567/jijap.53.067001

[18] Murase, K., Song, R. and Hiratsuka, S. (2014) Magnetic Particle Imaging of Blood Coagulation. Applied Physics Letters, 104, Article ID: 252409. http://dx.doi.org/10.1063/1.4885146

[19] Nishimoto, K., Mimura, A., Aoki, M., Banura, N. and Murase, K. (2015) Application of Magnetic Particle Imaging to Pulmonary Imaging Using Nebulized Magnetic Nanoparticles. Open Journal of Medical Imaging, 5, 49-55. http://dx.doi.org/10.4236/ojmi.2015.52008

[20] Murase, K., Mimura, A., Banura, N., Nishimoto, K. and Takata, H. (2015) Visualization of Magnetic Nanofibers Using Magnetic Particle Imaging. Open Journal of Medical Imaging, 5, 56-65. http://dx.doi.org/10.4236/ojmi.2015.52009

[21] Murase, K., Banura, N., Mimura, A. and Nishimoto, K. (2015) Simple and Practical Method for Correcting the Inhomogeneous Sensitivity of a Receiving Coil in Magnetic Particle Imaging. Japanese Journal of Applied Physics, 54, Article ID: 038001. http://dx.doi.org/10.7567/JJAP.54.038001

[22] Murase, K., Oonoki, J., Takata, H., Song, R., Angraini, A., Ausanai, P. and Matsushita, T. (2011) Simulation and Experimental Studies on Magnetic Hyperthermia with Use of Superparamagnetic Iron Oxide Nanoparticles. Radiological Physics and Technology, 4, 194-202. http://dx.doi.org/10.1007/s12194-011-0123-4

[23] Biederer, S., Knopp, T., Sattel, T.F., Ludtke-Buzug, K., Gleich, B., Weizenecker, J., Borgert, J. and Buzug, T.M. (2009) Magnetization Response Spectroscopy of Superparamagnetic Nanoparticles for Magnetic Particle Imaging. Journal of Physics D: Applied Physics, 42, Article ID: 205007. http://dx.doi.org/10.1088/0022-3727/42/20/205007

[24] Markov, D.E., Boeve, H., Gleich, B., Borgert, J., Antonelli, A., Sfara, C. and Magnani, M. (2010) Human Erythrocytes as Nanoparticle Carriers for Magnetic Particle Imaging. Physics in Medicine and Biology, 55, 6461-6473. http://dx.doi.org/10.1088/0031-9155/55/21/008

[25] Kallumadil, M., Tada, M., Nakagawa, T., Abe, M., Southern, P. and Pankhurst, Q.A. (2009) Suitability of Commercial Colloids for Magnetic Hyperthermia. Journal of Magnetism and Magnetic Materials, 321, 1509-1513. http://dx.doi.org/10.1016/j.jmmm.2009.02.075

[26] Box, G.E.P. and Lucas, H.L. (1959) Design of Experiments in Nonlinear Situations. Biometrika, 46, 77-90. http://dx.doi.org/10.1093/biomet/46.1-2.77

[27] Rodrigues, H.F., Mell, F.M., Branquinho, L.C., Zufelato, N., Silveira-Lacerda, E.P. and Bakuzis, A.F. (2013) RealTime Infrared Thermography Detection of Magnetic Nanoparticle Hyperthermia in a Murine Model under a NonUniform Field Configuration. International Journal of Hyperthermia, 29, 752-767. http://dx.doi.org/10.3109/02656736.2013.839056

[28] Andreu, I. and Natividad, E. (2013) Accuracy of Available Methods for Quantifying the Heat Power Generation of Nanoparticles for Magnetic Hyperthermia. International Journal of Hyperthermia, 29, 739-751. http://dx.doi.org/10.3109/02656736.2013.826825

[29] Shinkai, M., Yanase, M., Honda, H., Wakabayashi, T., Yoshida, J. and Kobayashi, T. (1996) Intracellular Hyperthermia for Cancer Using Magnetite Cationic Liposomes: In Vitro Study. Japanese Journal of Cancer Research, 87, 11791183. http://dx.doi.org/10.1111/j.1349-7006.1996.tb03129.x

[30] Le, B., Shinkai, M., Kitade, T., Honda, H., Yoshida, J., Wakabayashi, T. and Kobayashi, T. (2001) Preparation of Tumor-Specific Magnetoliposomes and Their Application for Hyperthermia. Journal of Chemical Engineering of Japan, 34, 66-72. http://dx.doi.org/10.1252/jcej.34.66

[31] Shinkai, M., Le, B., Honda, H., Yoshikawa, K., Shimizu, K., Saga, S., Wakabayashi, T., Yoshida, J. and Kobayashi, T. (2001) Targeting Hyperthermia for Renal Cell Carcinoma Using Human MN Antigen-specific Magnetoliposomes. Japanese Journal of Cancer Research, 92, 1138-1145. http://dx.doi.org/10.1111/j.1349-7006.2001.tb01070.x

[32] Suzuki, M., Shinkai, M., Kamihira, M. and Kobayashi, T. (1995) Preparation and Characteristics of Magnetite-Labelled Antibody with the Use of Poly (Ethylene Glycol) Derivatives. Biotechnology and Applied Biochemistry, 21, 335-345.

[33] Moroz, P., Jones, S.K., Winter, J. and Gray, B.N. (2001) Targeting Liver Tumors with Hyperthermia: Ferromagnetic Embolization in a Rabbit Liver Tumor Model. Journal of Surgical Oncology, 78, 22-29. http://dx.doi.org/10.1002/jso.1118

[34] Jordan, A., Scholz, R., Wust, P., Fahling, H., Krause, J., Wlodarczyk, W., Sander, B., Vogl, T. and Felix, R. (1997) Effects of Magnetic Fluid Hyperthermia (MFH) on $\mathrm{C} 3 \mathrm{H}$ Mammary Carcinoma in Vivo. International Journal of Hyperthermia, 13, 587-605. http://dx.doi.org/10.3109/02656739709023559

[35] Deng, Z.S. and Liu, J. (2002) Analytical Study on Bioheat Transfer Problems with Spatial or Transient Heating on Skin Surface or inside Biological Bodies. Journal of Biomechanical Engineering, 124, 638-649. 
http://dx.doi.org/10.1115/1.1516810

[36] Pennes, H.H. (1998) Analysis of Tissue and Arterial Blood Temperatures in the Resting Human Forearm. Journal of Applied Physiology, 85, 5-34.

[37] Shih, T.C., Kou, H.S., Liauh, C.T. and Lin, W.L. (2005) The Impact of Thermal Wave Characteristics on Thermal Dose Distribution during Thermal Therapy: A Numerical Study. Medical Physics, 32, 3029-3036. http://dx.doi.org/10.1118/1.2008507

[38] Skeete, Z., Cheng, H., Crew, E., Lin, L., Zhao, W., Joseph, P., Shan, S., Cronk, H., Luo, J., Li, Y., Zhang, Q. and Zhong, C.J. (2014) Design of Functional Nanoparticles and Assemblies for Theranostic Applications. ACS Applied Materials and Interfaces, 6, 21752-21768. http://dx.doi.org/10.1021/am502693t

[39] Murase, K., Takata, H., Takeuchi, Y. and Saito, S. (2013) Control of the Temperature Rise in Magnetic Hyperthermia with Use of a Static Magnetic Field. Physica Medica, 29, 624-630. http://dx.doi.org/10.1016/j.ejmp.2012.08.005

[40] Atsumi, T., Jeyadevan, B., Sato, Y. and Tohji, K. (2007) Heating Efficiency of Magnetite Particles Exposed to AC Magnetic Field. Journal of Magnetism and Magnetic Materials, 310, 2841-2843. http://dx.doi.org/10.1016/j.jmmm.2006.11.063

[41] Hergt, R., Hiergeist, R., Hilger, I., Kaiser, W.A., Lapatnikov, Y., Margel, S. and Richter, U. (2004) Maghemite Nanoparticles with Very High AC-Losses for Application in RF-Magnetic Hyperthermia. Journal of Magnetism and Magnetic Materials, 270, 345-357. http://dx.doi.org/10.1016/j.jmmm.2003.09.001

[42] Brezovich, I.A. and Meredith, R.F. (1989) Practical Aspects of Ferromagnetic Thermoseed Hyperthermia. Radiologic Clinics of North America, 27, 589-602.

[43] Hergt, R. and Dutz, S. (2007) Magnetic Particle Hyperthermia-Biophysical Limitations of a Visionary Tumour Therapy. Journal of Magnetism and Magnetic Materials, 311, 187-192. http://dx.doi.org/10.1016/j.jmmm.2006.10.1156

[44] Robson, M.D., Gatehouse, P.D., Bydder, M. and Bydder, G.M. (2003) Magnetic Resonance: An Introduction to Ultrashort TE (UTE) Imaging. Journal of Computer Assisted Tomography, 27, 825-846. http://dx.doi.org/10.1097/00004728-200311000-00001

[45] Idiyatullin, D., Corum, C., Park, J.Y. and Garwood, M. (2006) Fast and Quiet MRI Using a Swept Radiofrequency. Journal of Magnetic Resonance, 181, 342-349. http://dx.doi.org/10.1016/j.jmr.2006.05.014

[46] Zhang, J., Chamberlain, R., Etheridge, M., Idiyatullin, D., Corum, C., Bischof, J. and Garwood, M. (2014) Quantifying Iron-Oxide Nanoparticles at High Concentration Based on Longitudinal Relaxation Using a Three-Dimensional SWIFT Look-Locker Sequence. Magnetic Resonance in Medicine, 71, 1982-1988. http://dx.doi.org/10.1002/mrm.25181

[47] Hoopes, P.J., Petryk, A.A., Tate, J.A., Savellano, M.S., Strawbridge, R.R., Giustini, A.J., Stan, R.V., Gimi, B. and Garwood, M. (2013) Imaging and Modification of the Tumor Vascular Barrier for Improvement in Magnetic Nanoparticle Uptake and Hyperthermia Treatment Efficacy. Proceedings of SPIE Energy-Based Treatment of Tissue and Assessment VII, 8584, Article ID: 858403. http://dx.doi.org/10.1117/12.2008689 